\title{
Utility of Pulmonary Artery Acceleration Time to Estimate Systolic Pulmonary Artery Pressure in Neonates and Young Infants
}

\author{
Bassel Mohammad Nijres ${ }^{1,2}$ (1) $\cdot$ John Bokowski ${ }^{1}$ Lamya Mubayed ${ }^{1} \cdot$ Sabih H. Jafri ${ }^{1,3} \cdot$ Alan T. Davis ${ }^{4} \cdot$ Ra-id Abdulla $^{1,5}$
}

Received: 10 July 2019 / Accepted: 2 November 2019 / Published online: 9 November 2019

○) Springer Science+Business Media, LLC, part of Springer Nature 2019

\begin{abstract}
Transthoracic echocardiogram (TTE) is commonly used to screen for pulmonary hypertension (PHTN) in neonates and young infants. However, in the absence of sufficient tricuspid regurgitation (TR), a ventricular septal defect (VSD), or a patent ductus arteriosus (PDA), the estimation of systolic pulmonary artery pressure (SPAP) becomes challenging. Pulmonary artery acceleration time (PAAT) is an alternate parameter that is easy to obtain in almost all patients and does not require the presence of tricuspid valvar regurgitation or an anatomical cardiac defect. We sought to examine the correlation of PAAT with estimated SPAP by TTE and create an equation to estimate the SPAP using PAAT. We performed a retrospective review of TTEs performed on neonates and young infants (4 months of age or younger) at our institution between April 2017 and December 2018, along with the corresponding medical records. We included TTEs that provided estimation for SPAP and at least one PAAT measurement. During the study period, 138 TTEs performed on 82 patients met the inclusion criteria. Strong correlation was delineated between PAAT and SPAP estimated by the maximum velocity of tricuspid valve regurgitation Doppler, correlation coefficient $(r)=-0.83$. Moderate correlation was detected between PAAT and SPAP estimated by PDA Doppler, $r=-0.66$. Utilizing the following equation "SPAP $=82.6-0.58 \times \mathrm{PAAT}+\mathrm{RA}$ mean pressure", PAAT can be used to estimate SPAP in neonates and young infants. PAAT can be used as an alternative to TR jet to assess SPAP when the latter is absent or insufficient. Further studies are needed to verify the accuracy of this equation.
\end{abstract}

Keywords Pulmonary hypertension · Transthoracic echocardiogram · Pulmonary artery acceleration time

\section{Introduction}

Pulmonary hypertension (PHTN) is a commonly encountered problem in neonatal intensive care units (NICU) [1,2]. Right heart catheterization (RHC) remains the gold standard to diagnose PHTN. However, cardiac catheterization is an invasive procedure and carries substantial risk of complications [3-6]. In clinical practice, a transthoracic echocardiogram (TTE) is regularly ordered to screen for PHTN. Nevertheless, conventional TTE methods to screen for PHTN and assess systolic pulmonary artery pressure (SPAP) rely on the presence of sufficient tricuspid regurgitation (TR) or the presence of an anatomical cardiac defect (e.g., ventricular septal defect (VSD) and/or patent ductus arteriosus (PDA)). Therefore, without these factors, TTE assessment of SPAP in infants cannot be performed [7-12]. Pulmonary artery acceleration time (PAAT) is a promising alternative tool for noninvasive estimation of SPAP. It is easy to obtain and doesn't require presence of TR or an anatomical cardiac defect. PAAT correlates inversely with the SPAP, mean pulmonary 
artery pressure and calculated pulmonary vascular resistance (PVR). Moreover, in the adult literature, PAAT-based equations have been created to estimate pulmonary artery pressure $[11,13,14]$. To our knowledge, only one previous study performed in children utilized PAAT to estimate SPAP by creating an equation derived from linear regression analysis [15]. No such equations have been purposed to assess SPAP exclusively in neonates and young infants who are historically at higher risk of developing cardiac catheterization related complications (CCRC) [6].

Our study aimed to examine the correlation of PAAT with the estimated SPAP via TTE and to create a PAAT-based equation to estimate SPAP in neonates and young infants when traditional TTE methods cannot provide it.

\section{Material and Methods}

Institutional board review approval was obtained prior to conducting the study. This was a retrospective study reviewing medical records and echocardiographic data of young infants in the NICU and nursery at Rush University Children's Hospital.

We included patients in our NICU and nursery with a chronological age of $0-4$ months who had clinically indicated TTE from 04/01/2017-12/31/2018. We excluded patients in whom PAAT was not performed, patients with right ventricular outflow tract obstruction, and those with no other method to assess SPAP (e.g., patients with absent or insufficient TR, PDA and VSD Doppler forms). Collected data were stored on password protected computer on the REDCap website hosted by Rush University Medical Center.

\section{Echocardiography Analysis}

All TTEs were performed using an EPIQ7 machine (Phillips Medical Systems, USA, Andover, MA 01810). PAAT was obtained by placing the cursor in the center of the distal right ventricular outflow tract just below the pulmonary valve annulus. This position reduces the contamination of the doppler signal by the PDA flow at the MPA. In order to produce better flow characteristics and improve measurement accuracy, pulsed wave sweep speed was set on the max and the gain was adjusted to produce a clean uniform Doppler profile. Then, PAAT was measured from the resulting envelope (Fig. 1).

When more than one measurement of PAAT was obtained, the results were averaged. In our practice, systemic blood pressure (BP) is usually checked at the time of performing TTE, and BP measurement results and type of measurement (e.g., invasive versus non-invasive) are recorded. We used recorded blood pressure to estimate systolic pulmonary artery pressure when a good PDA

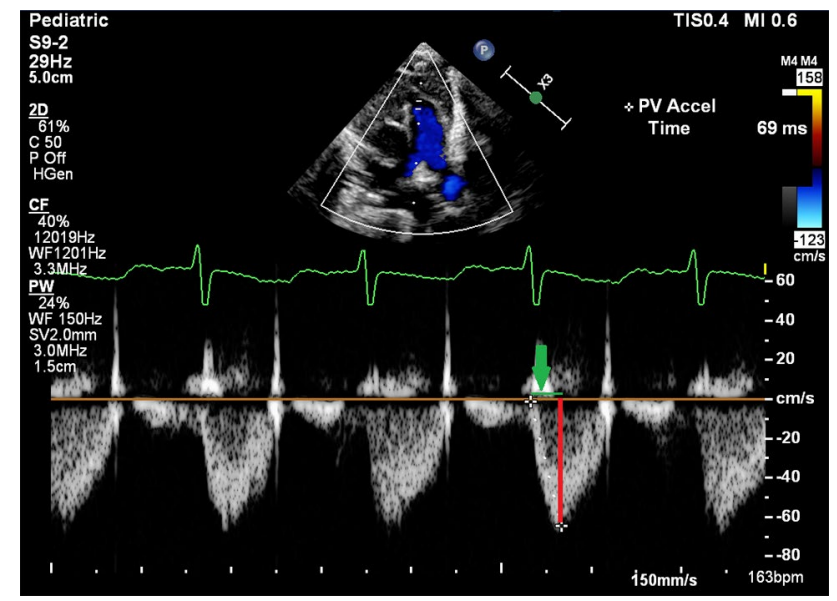

Fig. 1 A. Shows the Doppler cursor placed in the center of the pulmonary valve just proximal to the annulus with perfect alignment with the main pulmonary artery axis obtained from the parasternal short axis view. PAAT (the green line that depicted by the green arrow) is defined by the time between the onset of right ventricle ejection and peak flow velocity

and/or VSD Doppler were recorded. To further test the strength of PAAT with the different TTE methods used to estimate SPAP, we divided patients into three groups. Group 1: systolic pulmonary artery pressure estimated through maximum velocity of tricuspid valve regurgitation (TRVmax), group II: SPAP estimated based on the peak gradient across the VSD, and group III: SPAP estimated based on the peak gradient across the PDA. To investigate whether adjusting to heart rate (HR) would increase the strength of correlations, we divided PAAT by the square root of the RR interval [16]. Because pulmonary pressure and systemic artery pressure are dynamic and consistently change, TTEs performed in the same patient at more than $24 \mathrm{~h}$ interval were included.

\section{Statistical Analysis}

All statistical analyses were performed Stata v.15.1 (StataCorp, College Station, TX). Nominal data were expressed as numbers and percentages. Numerical data were reported as median with range or mean \pm standard deviation (SD). Linear regression was used to determine the relationship between variables. Linear regression was also used to determine the differences between groups (e.g., mild/moderate bronchopulmonary dysplasia (BPD) vs. severe BPD). The regression analysis utilized clustering on the subjects to obtain robust standard errors to adjust for non-independent observations. We used the bivariate Pearson's correlation coefficient (Pearson's r) to delineate the linear correlation between two parameters and to create a PAAT-derived equation to estimate SPAP. Significance was assessed at $p<0.05$. 


\section{Results}

During the study period, 138 TTEs that fit the inclusion criteria were performed on 82 subjects, of which 35 (42.7\%) were females and 47 (57.3\%) males. Of the 138 TTEs, 21 (15.2\%) were performed on full term infants while 117 $(84.8 \%)$ were obtained in premature infants. At the time of TTE, median gestational age for the premature infants was 25.3 (range 23-36.3) weeks, median chronologic age was 13 (range 0-118) days, median weight 1700 (range 500-4800) g, median height 38.4 (range 26-55) cm, mean systolic blood pressure (SBP) was $65 \pm 14.6 \mathrm{~mm} \mathrm{Hg}$, diastolic blood pressure was $37.1 \pm 12.2 \mathrm{~mm} \mathrm{Hg}$, and mean HR was $151.1 \pm 18.8 \mathrm{bpm}$. Of note, in 11 TTEs $(8 \%)$ the blood pressure measurements were obtained invasively, whereas 127 (92\%) BP measurements were recorded non-invasively. All patients who had invasive BP measurements didn't have VSD or PDA. In this study 43 TTEs were performed on infants with a diagnosis of BPD. Table 1 summarizes demographic and clinical data.

With regards to conventional TTE findings, sufficient TR to estimate SPAP was present in only $19(13.8 \%)$ of the performed TTEs. PDA was present in $116(84.1 \%)$ of patients, only one of which did not show good Doppler waveform to estimate SPAP. VSD was present in 10 TTEs, 9 of which had good Doppler waveform to estimate SPAP. Mean PAAT

Table 1 Demographics and clinical data

\begin{tabular}{ll}
\hline & Number $(\%)$ \\
\hline Sex & \\
Male & $47 / 82(57.3 \%)$ \\
Female & $35 / 82(42.7 \%)$ \\
Term/preterm & \\
Full term & $21 / 138(15.2 \%)$ \\
Preterm & $117 / 138(84.8 \%)$ \\
On respiratory support & \\
Yes & $111 / 137(81.0 \%)$ \\
Ventilator & $70 / 137(63.1 \%)$ \\
CPAP/BIPAP & $28 / 137(25.2 \%)$ \\
NC $+\mathrm{O}_{2}$ & $10 / 137(9.0 \%)$ \\
NC $-\mathrm{O}_{2}$ & $3 / 137(2.7 \%)$ \\
No & $26 / 137(19.0 \%)$ \\
BPD & \\
Mild & $5 / 43(11.6 \%)$ \\
Moderate & $5 / 43(11.6 \%)$ \\
Severe & $33 / 43(76.7 \%)$ \\
\hline
\end{tabular}

$M$ male, $F$ female, $H T$ height, $W t$ weight, $B S A$ body surface area, $S B P$ systolic blood pressure, $D B P$ diastolic blood pressure, $H R$ heart rate, $C P A P$ continuous positive airway pressure, BIPAP bilevel positive airway pressure, $\mathrm{NC}+\mathrm{O}_{2}$ nasal canula with oxygen supplementation, $\mathrm{NC}-\mathrm{O}_{2}$ nasal cannula without oxygen supplementation in all TTEs was $71.9 \pm 22.4 \mathrm{~ms}$ while mean PAAT adjusted to HR was $113.5 \pm 35.2 \mathrm{~ms}$. TTEs performed on patients with severe BPD showed shorter PAAT when compared with those with mild or moderate BPD, $70.8 \pm 24.4 \mathrm{~ms}$ and $88.0 \pm 20.5 \mathrm{~ms}(p=0.051)$. Table 2 summarizes TTE findings.

PAAT showed strong negative linear correlation with the estimated SPAP by TRVmax, correlation coefficient $(r)=0.83, p$ value $<0.001$. Good linear negative correlation was detected between PAAT and estimated SPAP using PDA or any method (combined groups), $r=-0.66$ and -0.63 respectively, with $p$ value of $<0.001$. Fair correlation was detected between the PAAT and estimated SPAP via the VSD, $r-0.43$, though $p$ value didn't reach statistical significance cut-point (Table 3). No significant change in the correlation was obtained when adjusting for the HR (Table 4). The best equation to estimate SPAP using PAAT

Table 2 Transthoracic echocardiography data

\begin{tabular}{ll}
\hline & Number $(\%)$ \\
\hline Sufficient TR jet & \\
Yes & $19 / 138(13.8 \%)$ \\
No & $119 / 138(86.2 \%)$ \\
Presence of VSD & \\
Yes & $10 / 138(7.3 \%)$ \\
No & $128 / 138(92.7 \%)$ \\
Presence of PDA & \\
Yes & $116 / 138(84.1 \%)$ \\
L $\rightarrow$ R & $102 / 138(87.9 \%)$ \\
R $\rightarrow$ L & $4 / 138(3.5 \%)$ \\
L $=\mathrm{R}$ & $10 / 138(8.6 \%)$ \\
No & $22 / 138(15.9 \%)$ \\
PAAT & $71.9 \pm 22.4$ \\
PAAT adjusted to $\mathrm{HR}^{\mathrm{a}}$ & $113.5 \pm 35.2$ \\
\hline
\end{tabular}

$T R$ tricuspid regurgitation, PDA patent ductus arteriosus, $L \rightarrow R$ left to right shunt, $R \rightarrow L$ right to left shunt, $L=R$ bidirectional shunt, $P A A T$ pulmonary artery acceleration time, $H R$ heart rate

${ }^{\mathrm{a}}$ Mean $+\mathrm{SD}$

Table 3 Correlation of PAAT with SPAP estimated by different TTE methods

\begin{tabular}{lrllr}
\hline Method & Number of TTE & $\begin{array}{l}\text { Number of } \\
\text { patients }\end{array}$ & $r$ & $p$ value \\
\hline TRVmax & 19 & 15 & -0.83 & $<\mathbf{0 . 0 0 1}$ \\
PDA & 115 & 68 & -0.66 & $<\mathbf{0 . 0 0 1}$ \\
VSD & 9 & 9 & -0.43 & 0.128 \\
Any method & 137 & 81 & -0.63 & $<\mathbf{0 . 0 0 1}$ \\
\hline
\end{tabular}

TRVmax maximum velocity of tricuspid valve regurgitation, $P D A$ patent ductus arteriosus, VSD ventricular septal defect, $r$ correlation coefficient 
Table 4 Correlation of PAAT adjusted to heart rate with SPAP estimated by different TTE methods

\begin{tabular}{lrcll}
\hline Method & Number of TTE & $\begin{array}{l}\text { Number of } \\
\text { patients }\end{array}$ & $r$ & $p$ value \\
\hline TRVmax & 19 & 15 & -0.81 & $<\mathbf{0 . 0 0 1}$ \\
PDA & 115 & 68 & -0.69 & $<\mathbf{0 . 0 0 1}$ \\
VSD & 9 & 9 & -0.53 & $<0.53$ \\
Any method & 137 & 81 & -0.66 & $<\mathbf{0 . 0 0 1}$ \\
\hline
\end{tabular}

TRVmax maximum velocity of tricuspid valve regurgitation, $P D A$ patent ductus arteriosus, VSD ventricular septal defect, $r$ correlation coefficient

is $\mathrm{SPAP}=82.6-0.58 \times \mathrm{PAAT}+\mathrm{RA}$ mean pressure. This equation was derived from the strong correlation of PAAT and estimated SPAP by TRVmax.

\section{Discussion}

PHTN is a frequently encountered problem in the NICU. It may present acutely shortly after birth, such as in persistent pulmonary hypertension of the newborn (PPHTN) or more indolently as with PHTN associated with BPD [17-19]. Increased pulmonary arterial pressure and PVR resistance in infants with BPD have been described in multiple studies [18-23]. Cardiac catheterization remains the gold standard to establish the diagnosis of pulmonary hypertension. Unless there is contraindication to cardiac catheterization or the patient is severely ill requiring immediate initiation of therapy, it is required to perform right heart catheterization (with left heart catheterization in the initial evaluation) to confirm the diagnosis, evaluate the etiology, stratify the severity and determine acute vasodilator response (AVR). This approach helps in guiding medical therapy and provides individualized prognostic insight [21, 24, 25].

Moreover, after initiation of a new anti-PHTN treatment for few months, it is also recommended to repeat cardiac catheterization to assess the response to treatment and the progression of the disease [21]. Nevertheless, cardiac catheterization is not a risk-free procedure. In fact, patients with PHTN are a high-risk group for CCRC. Cardiac arrest is estimated to occur in $0.6-2 \%$ and the risk of a pulmonary hypertension crisis is nearly $5 \%$. In addition, children with PHTN, especially young infants, inherently have higher risk of CCRC when compared with adults who carry the same diagnosis [3-5, 26, 27]. With the increased survival of the VLBW premature infants who commonly develop PHTN and are at high risk for CCRC, reliable surveillance for PHTN is needed in order to promptly identify and treat PHTN [28-30].

Point of care bedside TTE is commonly ordered in young infants to screen for PHTN [2, 10, 24, 31]. Although TTE is not a replacement for RHC in diagnosing PHTN, it has a crucial role in guiding clinical decision making in sick infants. It provides valuable longitudinal and real time functional and hemodynamic information [31]. Unfortunately, conventional TTE cannot estimate SPAP accurately in a high number of infants, especially those with BPD [19, 30, 31]. Measuring continuous-wave Doppler of the TRVmax is the most popular TTE method to estimate SPAP non-invasively. Simply, applying the modified Bernoulli equation to the measured TRVmax and adding estimated mean right atrial pressure (which is usually $2-5 \mathrm{mmHg}$ ) allows the estimation of SPAP. TRVmax correlates strongly with the invasive SPAP measurements [30, 32-36]. However, this method requires sufficient TR to generate accurate Doppler wave form which is not present in all patients [10-12]. Furthermore, in young infants with a history of prematurity and BPD, sufficient TR jet to estimate the SPAP is detected only in $44 \%$ of the echocardiograms [19]. In our cohort, sufficient TR jet to estimate SPAP was identified in only 19 (13.8\%) of the 138 performed TTEs. Factors associated with BPD (e.g., pulmonary hyperinflation with expansion of the thoracic cage) and change of the position of the heart may affect TTE's ability to detect the TR jet. However, the exact mechanism is yet to be determined [37]. The presence of anatomical defects, such as VSD and PDA, allows the measurement of the gradient across the anatomical defect providing an alternative method for the estimation of SPAP. These methods are restricted only to a small subset of patients who actually have the anatomical defect. In addition, the estimation of SPAP using these methods requires knowledge of the systemic arterial pressure at the time of measuring the gradient $[7-9,30]$. In the absence of TR or anatomical defects that allow assessing the SPAP, other TTE findings, such as right ventricular hypertrophy and flattening of interventricular septum can be qualitative indicators of PHTN, even though these findings are neither sensitive nor specific. Thus, they cannot be used to categorize the severity of PHTN [30].

PAAT measurement is an attractive method to assess the SPAP. Multiple studies have shown that PAAT inversely correlates with the severity of PHTN and can be used to estimate systolic and mean pulmonary artery pressure. PAAT does not rely on the presence of an anatomic defect or TR. Therefore, it can be utilized in the vast majority of patients to assess the SPAP $[11,13,14]$.

PAAT (Fig. 1) is defined as the time from the onset to the time at which maximum flow velocity occurs $[7,38,39]$. PAAT and the ratio of PAAT to the right ventricular ejection time (RVET) have been shown to be inversely correlated to PAP measured invasively during cardiac catheterization $[15,40]$. Only few studies have described the inverse relationship of PAAT and PHTN in infancy [19, 41]. In our study, PAAT in infants with severe BPD was lower compared with that of infants with less severe $\operatorname{BPD}(p=0.051)$. 
The insignificant $\mathrm{P}$ value appears to be related to the small sample size. However, our findings are in concurrence with the findings of Levy et al. [41] who reported that infants born prematurely exhibit an abnormally low PAAT compared with age matched term infants at 1-year of corrected gestation age. Moreover, preterm children with BPD showed even lower PAAT compared with those with no BPD. These findings suggest higher PVR in preterm infants that is more pronounced in those with BPD [41]. Levy et al. did not provide a PAAT derived equation to estimate SPAP and did not include newborn infants [41]. Despite the knowledge of the inverse relationship of PAAT and SPAP, the exact relationship between these two parameters, i.e., how to estimate SPAP by having PAAT, has not been described in neonates and young infants.

In our study, a strong correlation was demonstrated between PAAT and SPAP estimated by the TR jet $(r=-0.83)$. An equation was created to help estimate SPAP in newborns and young infants: $\mathrm{SPAP}=82.6-0.58 \times \mathrm{PAAT}+\mathrm{RA}$ mean pressure. This equation can be applied in the vast majority of infants (except those with pulmonary valve abnormalities), and is especially helpful in the absence of TR, VSD, or PDA. To the best of our knowledge, our study is the first to estimate SPAP using PAAT exclusively in neonates and young infants.

Although our findings suggest a strong correlation between PAAT and estimated SPAP utilizing TRVmax, weaker correlations were found when SBP was used to calculate SPAP (e.g., estimated by the PDA jet or by the VSD gradient). This can probably be explained by the inaccuracy of BP measurements rather than a real limitation of PAAT. In critically ill children, blood pressure measured by cuff does not correlate well with invasively measured blood pressure and cannot replace it when knowing that accurate blood pressure is crucial for minute-to-minute management [42, 43]. Similarly, in premature infants, especially at the lower systemic blood pressure range, noninvasive blood pressure measurements do not correlate well with invasive measurements and tend to significantly over-estimate BP, falsely reassuring the providing team [44].

Therefore, we believe that in the absence of the invasive BP measurement, the severity of PHTN in relation to the systemic BP pressure should not be assessed. Rather, the estimated SPAP should be stated as an absolute number which can be obtained by TRVmax, or PAAT if TRVmax is absent or insufficient. Estimated SPAP using the VSD or PDA gradient without simultaneous invasive BP measurement should be interpreted cautiously. In these instances, PAAT would be more reliable as it does not require knowledge of SBP to estimate SPAP.

Older studies have suggested correction for HR when the HR is out of the range (60-100 beats/min) to improve the accuracy of PAAT [11, 14, 16, 45-47]. In our study, adjusting for the HR did not increase the strength of correlation which is in consistent with the most recent studies showing that correction for HR is unnecessary and does not significantly improve the accuracy of PAAT $[15,41]$.

\section{Limitations}

Despite the fact that SPAP has not been validated against invasive RHC, PAAT showed a strong correlation with the most reliable available TTE method to estimate SPAP, i.e., TRVmax, and proved helpful in assessing SPAP when sufficient TR jet is absent. We did not investigate whether adjusting to RVET would result in improvement of the correlation, given that our findings have showed a strong correlation between PAAT and TRVmax. Levy et al. have shown no significant improvement in the strength of correlation of PAAT when it was adjusted to RVET [41].

The equation proposed in this study to assess SPAP can only be used in young infants and it is unlikely to be accurate in older children. PAAT normally tends to prolong with aging [39], making the creation of a generalized equation to predict SPAP in all pediatric age groups impossible.

\section{Conclusion}

PAAT correlates strongly with SPAP estimated by TRVmax. Measurement of PAAT in young infants may allow the estimation of SPAP using the following equation: $\mathrm{SPAP}=82.6-0.58 \times \mathrm{PAAT}+\mathrm{RA}$ mean pressure. Utilizing the PAAT method to assess SPAP will further strengthen the role of TTE in the screening for PHTN in young infants. Further studies from different institutions are needed to validate our findings. Confirming the utility of PAAT for estimation of SPAP in young infants will provide an invaluable tool to that would aid in the evaluation, diagnosis, and follow-up of young infants with PHTN in whom non-invasive estimation of SPAP would otherwise not be possible.

Funding There is no funding associated with this study.

\section{Compliance with Ethical Standards}

Conflict of interest The authors declare that they have no conflicts of interest related to this study.

Ethical Approval This study was agreed to be conducted by Rush University Institutional Review Board. 


\section{References}

1. Gill AB, Weindling AM (1993) Pulmonary artery pressure changes in the very low birthweight infant developing chronic lung disease. Arch Dis Child 68:303-307

2. Gill AB, Weindling AM (1995) Raised pulmonary artery pressure in very low birthweight infants requiring supplemental oxygen at 36 weeks after conception. Arch Dis Child Fetal Neonatal Ed 72:F20-F22

3. Taylor CJ, Derrick G, McEwan A, Haworth SG, Sury MR (2007) Risk of cardiac catheterization under anaesthesia in children with pulmonary hypertension. Br J Anaesth 98:657-661

4. Carmosino MJ, Friesen RH, Doran A, Ivy DD (2007) Perioperative complications in children with pulmonary hypertension undergoing noncardiac surgery or cardiac catheterization. Anesth Analg 104:521-527

5. Williams GD, Maan H, Ramamoorthy C, Kamra K, Bratton SL, Bair E, Kuan CC, Hammer GB, Feinstein JA (2010) Perioperative complications in children with pulmonary hypertension undergoing general anesthesia with ketamine. Paediatr Anaesth 20:28-37

6. Jayaram N, Spertus JA, Kennedy KF, Vincent R, Martin GR, Curtis JP, Nykanen D, Moore PM, Bergersen L (2017) Modeling major adverse outcomes of pediatric and adult patients with congenital heart disease undergoing cardiac catheterization: observations from the NCDR IMPACT Registry (National Cardiovascular Data Registry Improving Pediatric and Adult Congenital Treatment). Circulation 136:2009-2019

7. Ge Z, Zhang Y, Kang W, Fan D, Ji X, Duran C (1993) Noninvasive evaluation of right ventricular and pulmonary artery systolic pressures in patients with ventricular septal defects: simultaneous study of Doppler and catheterization data. Am Heart J 125:1073-1081

8. Marx GR, Allen HD, Goldberg SJ (1985) Doppler echocardiographic estimation of systolic pulmonary artery pressure in pediatric patients with interventricular communications. J Am Coll Cardiol 6:1132-1137

9. Musewe NN, Poppe D, Smallhorn JF, Hellman J, Whyte H, Smith B, Freedom RM (1990) Doppler echocardiographic measurement of pulmonary artery pressure from ductal Doppler velocities in the newborn. J Am Coll Cardiol 15:446-456

10. Bhat R, Salas AA, Foster C, Carlo WA, Ambalavanan N (2012) Prospective analysis of pulmonary hypertension in extremely low birth weight infants. Pediatrics 129:e682-e689

11. Yared K, Noseworthy P, Weyman AE, McCabe E, Picard MH, Baggish AL (2011) Pulmonary artery acceleration time provides an accurate estimate of systolic pulmonary arterial pressure during transthoracic echocardiography. J Am Soc Echocardiogr 24:687-692

12. Hinderliter AL, Willis PW 4th, Long WA, Clarke WR, Ralph D, Caldwell EJ, Williams W, Ettinger NA, Hill NS, Summer WR, de Boisblanc B, Koch G, Li S, Clayton LM, Jobsis, MM, Crow JW, PPH Study Group (2003) Frequency and severity of tricuspid regurgitation determined by Doppler echocardiography in primary pulmonary hypertension. Am J Cardiol 91(1033-7):A9

13. Cowie B, Kluger R, Rex S, Missant C (2016) The relationship between pulmonary artery acceleration time and mean pulmonary artery pressure in patients undergoing cardiac surgery: An observational study. Eur J Anaesthesiol 33:28-33

14. Kitabatake A, Inoue M, Asao M, Masuyama T, Tanouchi J, Morita T, Mishima M, Uematsu M, Shimazu T, Hori M, Abe H (1983) Noninvasive evaluation of pulmonary hypertension by a pulsed Doppler technique. Circulation 68:302-309

15. Levy PT, Patel MD, Groh G, Choudhry S, Murphy J, Holland MR, Hamvas A, Grady MR, Singh GK (2016) Pulmonary artery acceleration time provides a reliable estimate of invasive pulmonary hemodynamics in children. J Am Soc Echocardiogr 29:1056-1065

16. Kawahito S, Kitahata H, Tanaka K, Nozaki J, Oshita S (2001) Pulmonary arterial pressure can be estimated by transesophageal pulsed doppler echocardiography. Anesth Analg 92:1364-1369

17. Steurer MA, Jelliffe-Pawlowski LL, Baer RJ, Partridge JC, Rogers EE, Keller RL (2017) Persistent Pulmonary Hypertension of the Newborn in Late Preterm and Term Infants in California. Pediatrics. https://doi.org/10.1542/peds.2016-1165

18. Fitzgerald D, Evans N, Van Asperen P, Henderson-Smart D (1994) Subclinical persisting pulmonary hypertension in chronic neonatal lung disease. Arch Dis Child Fetal Neonatal Ed 70:F118-F122

19. Benatar A, Clarke J, Silverman M (1995) Pulmonary hypertension in infants with chronic lung disease: non-invasive evaluation and short term effect of oxygen treatment. Arch Dis Child Fetal Neonatal Ed 72:F14-F19

20. Farstad T, Brockmeier F, Bratlid D (1995) Cardiopulmonary function in premature infants with bronchopulmonary dysplasiaa 2-year follow up. Eur J Pediatr 154:853-858

21. Abman SH, Wolfe RR, Accurso FJ, Koops BL, Bowman CM, Wiggins JW Jr (1985) Pulmonary vascular response to oxygen in infants with severe bronchopulmonary dysplasia. Pediatrics 75:80-84

22. Berman W Jr, Yabek SM, Dillon T, Burstein R, Corlew S (1982) Evaluation of infants with bronchopulmonary dysplasia using cardiac catheterization. Pediatrics 70:708-712

23. Goodman G, Perkin RM, Anas NG, Sperling DR, Hicks DA, Rowen M (1988) Pulmonary hypertension in infants with bronchopulmonary dysplasia. J Pediatr 112:67-72

24. Douwes JM, van Loon RL, Hoendermis ES, Vonk-Noordegraaf A, Roofthooft MT, Talsma MD, Hillege HL, Berger RM (2011) Acute pulmonary vasodilator response in paediatric and adult pulmonary arterial hypertension: occurrence and prognostic value when comparing three response criteria. Eur Heart J 32:3137-3146

25. Apitz C, Hansmann G, Schranz D (2016) Hemodynamic assessment and acute pulmonary vasoreactivity testing in the evaluation of children with pulmonary vascular disease. Expert consensus statement on the diagnosis and treatment of paediatric pulmonary hypertension. The European Paediatric Pulmonary Vascular Disease Network, endorsed by ISHLT and DGPK. Heart 102 Suppl 2: ii23-9.

26. Beghetti M, Schulze-Neick I, Berger RM, Ivy DD, Bonnet D, Weintraub RG, Saji T, Yung D, Mallory GB, Geiger R, Berger JT, Barst RJ, Humpl T, Investigators TOPP (2016) Haemodynamic characterisation and heart catheterisation complications in children with pulmonary hypertension: Insights from the Global TOPP Registry (tracking outcomes and practice in paediatric pulmonary hypertension). Int J Cardiol 203:325-330

27. O’Byrne ML, Kennedy KF, Kanter JP, Berger JT, Glatz AC (2018) Risk Factors for major early adverse events related to cardiac catheterization in children and young adults with pulmonary hypertension: an analysis of data from the IMPACT (Improving Adult and Congenital Treatment) Registry. J Am Heart Assoc. https://doi. org/10.1161/JAHA.117.008142

28. Stoll BJ, Hansen NI, Bell EF, Shankaran, S, Laptook, AR, Walsh MC, Hale EC, Newman NS, Schibler, K, Carlo WA, Kennedy KA, Poindexter BB, Finer NN, Ehrenkranz, RA, Duara, S, Sanchez PJ, O'Shea TM, Goldberg RN, Van, Meurs, KP, Faix, RG, Phelps DL, Frantz ID,3rd, Watterberg, KL, Saha, S, Das A, Higgins RD, Eunice Kennedy Shriver National Institute of Child Health, and Human Development Neonatal Research Network (2010) Neonatal outcomes of extremely preterm infants from the NICHD Neonatal Research Network. Pediatrics 126:443-456 
29. Subhedar NV, Shaw NJ (2000) Changes in pulmonary arterial pressure in preterm infants with chronic lung disease. Arch Dis Child Fetal Neonatal Ed 82:F243-F247

30. Nagiub M, Lee S, Guglani L (2015) Echocardiographic assessment of pulmonary hypertension in infants with bronchopulmonary dysplasia: systematic review of literature and a proposed algorithm for assessment. Echocardiography 32:819-833

31. Mourani PM, Sontag MK, Younoszai A, Ivy DD, Abman SH (2008) Clinical utility of echocardiography for the diagnosis and management of pulmonary vascular disease in young children with chronic lung disease. Pediatrics 121:317-325

32. Burnard ED, James LS (1963) Atrial pressures and cardiac size in the newborn infant. Relationships with degree of birth asphyxia and size of placental transfusion. J Pediatr 62:815-826

33. Zellers T, Gutgesell HP (1989) Noninvasive estimation of pulmonary artery pressure. J Pediatr 114:735-741

34. Yock PG, Popp RL (1984) Noninvasive estimation of right ventricular systolic pressure by Doppler ultrasound in patients with tricuspid regurgitation. Circulation 70:657-662

35. Amsallem M, Sternbach JM, Adigopula S, Kobayashi Y, Vu TA, Zamanian R, Liang D, Dhillon G, Schnittger I, McConnell MV, Haddad F (2016) Addressing the Controversy of Estimating Pulmonary Arterial Pressure by Echocardiography. J Am Soc Echocardiogr 29:93-102

36. van Riel AC, Opotowsky AR, Santos M, Rivero JM, Dhimitri A, Mulder BJ, Bouma BJ, Landzberg MJ, Waxman AB, Systrom DM, Shah AM (2017) Accuracy of echocardiography to estimate pulmonary artery pressures with exercise: a simultaneous invasive-noninvasive comparison. Circ Cardiovasc Imaging. https:// doi.org/10.1161/CIRCIMAGING.116.005711

37. Arcasoy SM, Christie JD, Ferrari VA, Sutton MS, Zisman DA, Blumenthal NP, Pochettino A, Kotloff RM (2003) Echocardiographic assessment of pulmonary hypertension in patients with advanced lung disease. Am J Respir Crit Care Med 167:735-740

38. Haddad F, Zamanian R, Beraud AS, Schnittger I, Feinstein J, Peterson T, Yang P, Doyle R, Rosenthal D (2009) A novel noninvasive method of estimating pulmonary vascular resistance in patients with pulmonary arterial hypertension. J Am Soc Echocardiogr 22:523-529

39. Koestenberger M, Grangl G, Avian A, Gamillscheg A, Grillitsch M, Cvirn G, Burmas A, Hansmann G (2017) Normal reference values and $\mathrm{z}$ scores of the pulmonary artery acceleration time in children and its importance for the assessment of pulmonary hypertension. Circ Cardiovasc Imaging. https://doi.org/10.1161/ CIRCIMAGING.116.005336

40. Kosturakis D, Goldberg SJ, Allen HD, Loeber C (1984) Doppler echocardiographic prediction of pulmonary arterial hypertension in congenital heart disease. Am J Cardiol 53:1110-1115

41. Levy PT, Patel MD, Choudhry S, Hamvas A, Singh GK (2018) Evidence of echocardiographic markers of pulmonary vascular disease in asymptomatic infants born preterm at one year of age. J Pediatr 197:48-56.e2

42. Cambiaso-Daniel J, Rontoyanni VG, Foncerrada G, Nguyen A, Capek KD, Wurzer P, Lee JO, Hundeshagen G, Voigt CD, Branski LK, Finnerty CC, Herndon DN (2018) Correlation between invasive and noninvasive blood pressure measurements in severely burned children. Burns 44:1787-1791

43. Joffe R, Duff J, Garcia Guerra G, Pugh J, Joffe AR (2016) The accuracy of blood pressure measured by arterial line and non-invasive cuff in critically ill children. Crit Care 20: 177-016-1354-x.

44. Takci S, Yigit S, Korkmaz A, Yurdakok M (2012) Comparison between oscillometric and invasive blood pressure measurements in critically ill premature infants. Acta Paediatr 101:132-135

45. Dabestani A, Mahan G, Gardin JM, Takenaka K, Burn C, Allfie A, Henry WL (1987) Evaluation of pulmonary artery pressure and resistance by pulsed Doppler echocardiography. Am J Cardiol 59:662-668

46. Tossavainen E, Soderberg S, Gronlund C, Gonzalez M, Henein MY, Lindqvist P (2013) Pulmonary artery acceleration time in identifying pulmonary hypertension patients with raised pulmonary vascular resistance. Eur Heart J Cardiovasc Imaging 14:890-897

47. Chan KL, Currie PJ, Seward JB, Hagler DJ, Mair DD, Tajik AJ (1987) Comparison of three Doppler ultrasound methods in the prediction of pulmonary artery pressure. J Am Coll Cardiol 9:549-554

Publisher's Note Springer Nature remains neutral with regard to jurisdictional claims in published maps and institutional affiliations. 